\title{
HEPATIC ENZYMES CHANGES IN CHRONIC KIDNEY DISEASE PATIENTS- A NEED FOR MODIFIED REFERENCE VALUES
}

Omar B. Latiwesh'1, Mustafa Y. G. Younis², Srikumar Shakila³, Fatima Abdulmalik4, Jamal A. Alammari5, Yupa Min', Azhar Hussain7, Jagannadha Rao Peela ${ }^{8}$

${ }_{1}^{1}$ Faculty of Medicine, Department of Laboratory Medicine, High Institute of Medical Technology, Benghazi, Libya.

${ }^{2}$ Faculty of Medicine, Assistant Professor and HOD of Biochemistry, University of Benghazi, Benghazi, Libya.

${ }^{3}$ Faculty of Medicine, Department of Biochemistry, Quest International University Perak, IPOH, Malaysia.

${ }^{4}$ Faculty of Medicine, Department of Biochemistry, High Institute of Medical Technology, Benghazi, Libya.

${ }_{5}^{5}$ Faculty of Medicine, Department of Laboratory Medicine, High Institute for Comprehensive Vocations-Gamins, Libya.

${ }^{6}$ Faculty of Medicine, Department of Pathology, Quest International University Perak, IPOH, Malaysia.

${ }^{7}$ Research Scholar, Department of Medicine, St. Matthew's University School of Medicine, Cayman Island.

${ }^{8}$ Faculty of Medicine, Associate Professor at the Department of Medical Genetics and Biochemistry, St. Matthew's University

School of Medicine, Cayman Island.

\section{ABSTRACT}

\section{BACKGROUND}

Aspartate aminotransferase (AST), alanine aminotransferase (ALT) and alkaline phosphatase (ALP) are markers of aggression to hepatocytes and aid in the diagnosis, monitoring and treatment of liver diseases because they reflect the inflammatory activity of the liver. The present study aimed to estimate the changes in serum ALT, AST and ALP levels in both pre-dialysis (pre-HDCKD) and haemodialysis (HD-CKD) patients in comparison to healthy controls, and to find out the association between hepatic enzyme changes and estimated Glomerular Filtration Rate (eGFR), body weight loss, duration of disease and duration of haemodialysis in CKD patients.

\section{MATERIALS AND METHODS}

The present descriptive comparative study conducted on 53 HD-CKD patients, 61 pre-HD-CKD patients and 50 healthy controls. The clinical information and medical history were obtained through the review of patient's medical files and patient's interviews. A face-to-face interview was conducted based on a questionnaire that included variables such as age, sex, date of the diagnosis, cause of the disease, blood pressure, height, weight, duration of haemodialysis and any health problems or prescriptions. Blood samples were collected from all the participants and analysed for urea, creatinine, AST, ALT and ALP using fully automated machines. The data were statistically analysed using SPSS software and p-value less than 0.05 was considered as statistically significant.

\section{RESULTS}

Our results revealed lower AST and ALT levels in HD-CKD patients than both pre-HD-CKD patients and healthy controls. On the contrary, the difference in aminotransferase level between pre-HD-CKD patients and control was statistically non-significant. Moreover, ALP was significantly higher in both groups of CKD patients when compared to healthy controls. In addition, a significant negative correlation has been shown between blood urea and aspartate aminotransferase level in pre-HD-CKD group.

\section{CONCLUSION}

Serum ALT and AST levels tend to be reduced in CKD patients, particularly in those on maintenance haemodialysis treatment, a finding that enforces the urgent need for the establishment of separate reference ranges of liver function tests for CKD patients in order to facilitate the diagnosis, monitoring and treatment of liver diseases, especially hepatitis $\mathrm{C}$ infection, an establish ment that may play a role in decreasing the mortality in the CKD patients.

\section{KEYWORDS}

Hepatic Enzymes, Chronic Kidney Disease, AST, ALT, ALP.

HOW TO CITE THIS ARTICLE: Latiwesh OB, Younis MYG, Shakila S, et al. Hepatic enzymes changes in chronic kidney disease patients- a need for modified reference values. J. Evolution Med. Dent. Sci. 2018;7(16):1949-1954, DOI: $10.14260 /$ jemds/2018/439

'Financial or Other Competing Interest': None.

Submission 21-03-2018, Peer Review 03-04-2018,

Acceptance 05-04-2018, Published 16-04-2018.

Corresponding Author:

Mustafa Y. G. Younis,

Assistant Professor,

HOD of Biochemistry,

University of Benghazi, Benghazi, Libya.

E-mail: jagannadh21@yahoo.co.uk

DOI: $10.14260 /$ jemds $/ 2018 / 439$

\section{BACKGROUND}

Chronic kidney disease (CKD) results from the inability of the kidneys to filter waste products (e.g. urea and creatinine) from the circulation. CKD is considered as a major health problem worldwide. ${ }^{1}$ Diabetes, hypertension and obesity are the most implicated risk factors for CKD in the Middle East. ${ }^{2}$ Chronic kidney disease (CKD), also known as renal failure can be reversible (often acute) or irreversible which is progressive decline in the glomerular filtration rate (GFR) to below $25 \%$ of the normal level (decline of $30 \mathrm{~mL} / \mathrm{min} / 1.73$ $\mathrm{m} 2$ ) for at least 3 months. ${ }^{3,4,5}$ 
Glomerular filtration rate (GFR) lower than $60 \mathrm{~mL} / \mathrm{min}$ per $1.73 \mathrm{~m} 2$ is the cut-off value for the diagnosis of CKD, which is usually presented with symptoms of uraemia. 3,6 Regardless of the cause of kidney damage, once a critical level of renal function deterioration is reached, progression to end-stage renal failure (ESRD) is inevitable. ESRD is a term reserved when haemodialysis or a kidney transplant is required. 5

ESRD incidence rate in Libya is one of the highest in the world. An estimation of 2417 adult ESRD patients are registered in Libya. The prevalence rate of ESKD undergoing haemodialysis was 624 per million population. ${ }^{6}$

The serum levels of the liver enzymes alanine aminotransferase (ALT), aspartate aminotransferase (AST) and alkaline phosphatase (ALP) are markers of aggression against hepatocytes. 7,8 The ALT was formerly known as serum glutamic-pyruvic transaminase (SGPT) and AST as serum glutamic-oxaloacetic transaminase (SGOT). Thus, they are elevated in several diseases such as chronic viral hepatitis, non-alcoholic fatty liver disease, autoimmune hepatitis, haemochromatosis and alcoholic liver disease. These enzymes assist in diagnosis, follow-up and response to treatment because they reflect inflammatory activity in liver parenchyma. ${ }^{8}$

The prevalence of hepatitis $\mathrm{C}$ virus (HCV) infection is significantly higher in haemodialysis (HD) patients than in the general population. ${ }^{9}$ This disease is associated with an increased mortality rate, primarily due to hepatocellular carcinoma and liver cirrhosis. ${ }^{10,11}$ The prevalence of HCV infection in HD patients varies greatly between regions of the world and even within the same country. ${ }^{12}$ The prevalence rates of hepatitis $\mathrm{C}$ are $31.1 \%$ for dialysis patients in Libya, $5.5 \%$ for dialysis patients in Brazil, $14.4 \%$ for those in the United States and $68 \%$ for those in Saudi Arabia.12,13,14,15 Although, HCV infection results in an increase in ALT, these levels are generally lower in haemodialysis patients. Interestingly, some studies have shown that patients with chronic kidney disease (CKD) on HD may have lower serum levels of liver enzymes than those with a normal renal function for reasons that remain unclear. ${ }^{16}$ This profile may adversely affect the diagnosis, clinical management and treatment of liver disease in these patients. It was hypothesised that the reduction in aminotransferase enzymes could be caused by several factors including elevated serum lactate concentration and deficiency of the vitamin cofactor involved in transaminase biosynthesis. ${ }^{17,18}$

On the other hand, plasma ALP levels can originate from liver, bone, intestine and placenta. In general, the isoenzymes from liver and bone contribute to the majority of the circulating enzyme levels. Therefore, in a patient with liver disease serum ALP level is an important marker for screening and monitoring. However, in a CKD patient, renal osteodystrophy could result in a significant increase in the bone isoenzyme of ALP contributing to high serum ALP level. In fact, higher ALP has been associated with increased mortality in pre-dialysis CKD as well as patients on maintenance haemodialysis. ${ }^{19}$

\section{Objectives}

The present study is the first of its kind in Libya and it was undertaken to estimate the changes in serum alanine aminotransferase, aspartate aminotransferase and alkaline phosphatase levels in both pre-dialysis and haemodialysis chronic kidney disease patients in comparison to normal healthy control, and to find out the association between hepatic enzyme changes and eGFR, body weight loss, duration of disease and duration of haemodialysis in CKD patients. 18

\section{MATERIALS AND METHODS}

\section{Subjects}

The present descriptive comparative study included 110 chronic kidney disease patients (53 patients were undergoing maintenance haemodialysis and 61 patients were in pre-dialysis stages) recruited from the Centre of Kidney Diseases Services- Benghazi and 50 age and sex-matched healthy controls selected from Al-Saleem LaboratoryBenghazi.

Informed consent was obtained from all participants before the study. The selection of patients was based on the previous diagnosis with chronic kidney disease and that diagnosis was dependent on KDIGO guidelines (CKD is defined as kidney damage marked by albuminuria and GFR less than $60 \mathrm{~mL} / \mathrm{min}$ per $1.73 \mathrm{~m} 2$ for $\leq 3$ months). ${ }^{20}$

Clinical information and medical history were obtained through the review of patient's medical files and patient's interviews. Face-to-face interview were based on a questionnaire that included variables such as age, sex, date of the diagnosis, cause of the disease, blood pressure, weight, height, duration of haemodialysis with exclusion of patients undergoing haemodialysis for less than 6 months, times of haemodialysis/ week, CKD treatments and any health problems or prescriptions.

Patients suffering from any disease other than CKD that could affect their metabolic status and the parameters studied such as malignancy, chronic viral hepatitis, nonalcoholic fatty liver disease, autoimmune hepatitis, haemochromatosis and alcoholic liver disease were excluded from the study. Pregnant and lactating women were excluded and patients with a history of recent surgery, smoking or alcohol intake were also excluded. The history of medication was recorded and patients taking any drugs that could affect liver function were excluded.

The control group consisted of non-alcoholic healthy subjects with no history of inherited or acquired kidney or liver diseases.

\section{Methods}

Venous blood samples were collected from all participants in plain tubes. Serum was obtained by centrifugation of clotted blood and stored at $-20^{\circ} \mathrm{C}$ until the assays were performed. The measurements of blood urea nitrogen (BUN), serum creatinine, alanine aminotransferase (ALT), aspartate aminotransferase (AST) and alkaline phosphatase (ALP) were done using an automated routine chemistry analyser (Roche Cobas Integra 400 plus, Roche Diagnostics Limited, Switzerland) with commercial kits (Roche Cobas packs, Roche Diagnostics Limited, Switzerland) according to manufacturer's protocol. 
Estimation of Glomerular Filtration Rate (eGFR):

The Glomerular Filtration Rate was estimated by using Cockcroft-Gault Formula 21

Men: $\frac{(140-\text { age in years) (weight in } \mathrm{Kg})}{72 \times \text { serum } \mathrm{Cr}}$

Women: $\frac{(140-\text { age in years) }(\text { weight in } \mathrm{Kg})}{72 \times \text { serum } \mathrm{Cr}} \times 0.8$

\section{Statistical Analysis}

The data were analysed using the statistical package for the social sciences (SPSS version 17). Descriptive characteristics of the study participants were calculated as the mean \pm standard deviation (SD). Analysis of Variance (ANOVA) was used to determine the differences in subject's characteristics. Pearson's correlation coefficient determination was done to evaluate the degree of association between hepatic enzymes changes and clinical and biochemical parameters. A p-value $<0.05$ was considered as statistically significant.

\section{RESULTS}

The mean age and standard deviation (SD) of the haemodialysed CKD patients selected for this study were $53.2 \pm 13$, and the male: female ratio was $5: 4$. The age range was 18 - 95 years with the duration of disease ranging from 1- 32 years and duration of haemodialysis ranged from 1- 16 years. The mean age and SD of the pre-dialysed CKD patients included in the study were $51.5 \pm 14.8$, and the male: female ratio was 1: 3 . The age range was $25-90$ years with the duration of disease ranged from 1 - 13 years. The mean age and SD of the control subjects was $47.4 \pm 12.4$, and the male:female ratio was 1: 3 . The age range was 19 - 67 years.

\section{Renal Function Test (RFT) (Blood Urea and Serum Creatinine)}

Blood urea and creatinine levels were significantly higher in pre-dialysed CKD (Pre-HD-CKD) patients than controls $(\mathrm{p}<0.05)$ (Table 1$)$.

\begin{tabular}{|c|c|c|c|}
\hline Parameters & $\begin{array}{c}\text { Pre-HD-CKD } \\
\text { Patients N=61 }\end{array}$ & $\begin{array}{c}\text { Controls } \\
\mathbf{N}=\mathbf{5 0}\end{array}$ & P value \\
\hline BUN (mg/dL) & $107.4 \pm 39.3$ & $25 \pm 8.7$ & $\mathrm{p}<0.05$ \\
\hline $\begin{array}{c}\text { S. creatinine } \\
\text { (mg/dL) }\end{array}$ & $3.78 \pm 2.3$ & $0.75 \pm 0.2$ & $\mathrm{p}<0.05$ \\
\hline
\end{tabular}

Table 1. Mean \pm SD of Blood Urea and Serum Creatinine in Pre-Dialysed CKD Patients and Control Subjects

\section{Aspartate Aminotransferase (AST)}

Haemodialysed CKD patients had a significantly lower AST concentration when compared to pre-dialysed CKD patients $(p=0.04)$, and normal healthy controls $(p=0.01)$. The difference in AST levels between pre-dialysed CKD patients and healthy controls was statistically non-significant $(p=0.6)$.

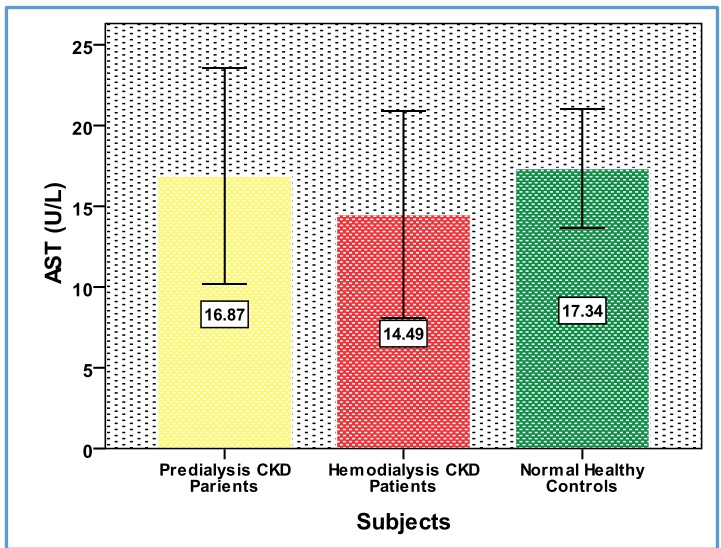

Figure 1. Mean $\pm S D(U / L)$ of Aspartate Aminotransferase (AST) in CKD Patients and Healthy Controls

Alanine Aminotransferase (ALT)

Alanine aminotransferase levels were significantly lower in haemodialysed CKD patients than pre-dialysed CKD patients $(p=0.00)$ and normal healthy controls $(p=0.00)$. No significant difference in ALT levels has been shown between pre-dialysed CKD patients and normal healthy controls ( $\mathrm{p}=$ 0.3) (Figure 2).

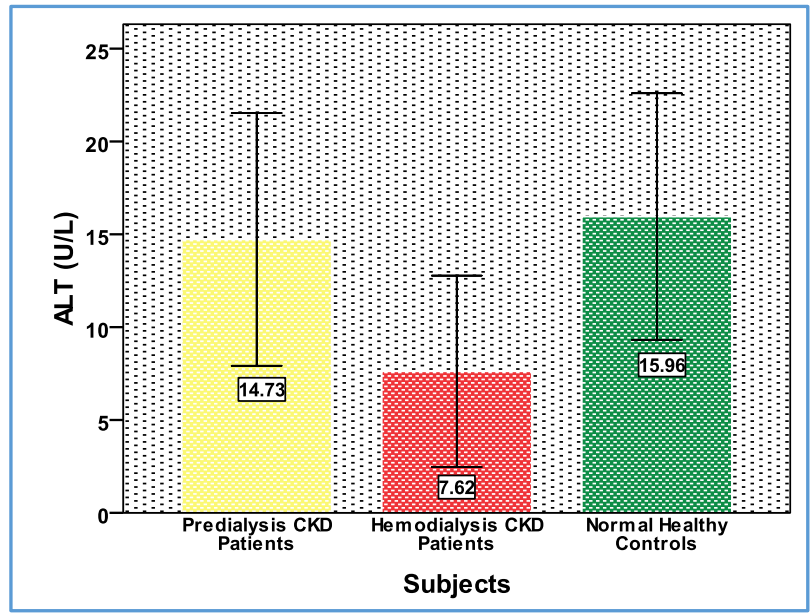

Figure 2. Mean $\pm S D(U / L)$ of Alanine Aminotransferase (ALT) in CKD Patients and Controls

\section{Alkaline Phosphatase (ALP)}

The mean level of alkaline phosphatase was significantly higher in both groups of CKD patients when compared to normal healthy controls (pre-dialysed CKD patients vs. controls $(p=0.00)$; haemodialysed CKD patients vs. controls $(p=0.04)$. Pre-dialysed CKD patients showed higher levels of ALP than haemodialysed CKD patients, and the difference was statistically significant $(p=0.01)$ (Figure 2$)$. 


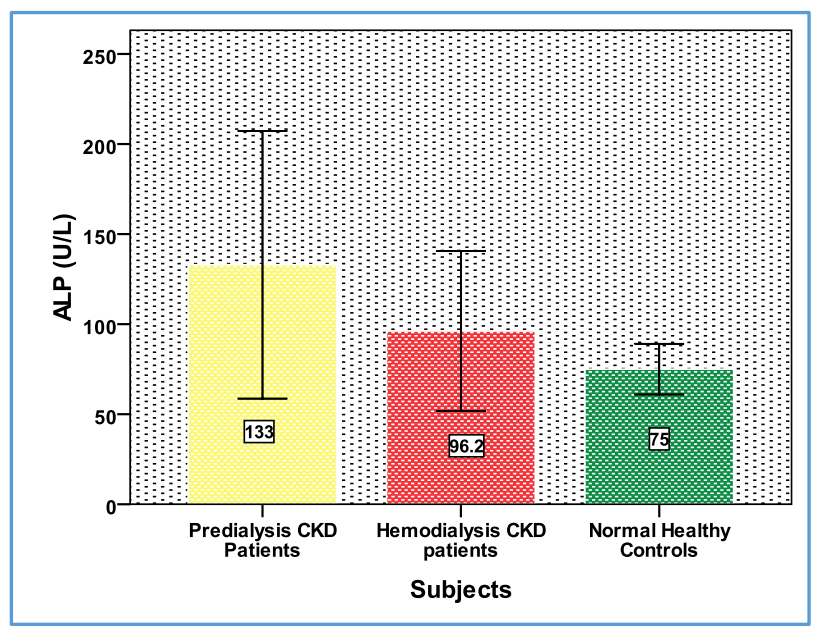

Figure 3. Mean $\pm S D(U / L)$ of Alkaline Phosphatase in CKD Patients and Control Subjects

\section{Correlations of Hepatic Enzymes Levels with Other Parameters}

There were non-significant correlations between hepatic enzymes changes (AST, ALT, ALP) and age, duration of disease or duration of haemodialysis in CKD patients. In predialysed CKD patients, AST showed a significant negative correlation with blood urea $(r=-0.285, p=0.03)$ (Figure 4), but non-significant correlation with serum creatinine $(r=-$ 0.076, $\mathrm{p}=0.5$ ) and eGFR ( $\mathrm{r}=0.04, \mathrm{p}=0.8)$. On the other hand, no significant correlations have been shown between either ALT or ALP and urea (ALT vs. urea ( $\mathrm{r}=-0.09, \mathrm{p}=0.5)$; ALP vs. urea $(\mathrm{r}=-0.14, \mathrm{p}=0.3)$, creatinine (ALT vs. creatinine $(\mathrm{r}=0.13$, $\mathrm{p}=0.33)$; ALP vs. creatinine $(\mathrm{r}=-0.19, \mathrm{p}=0.2)$ or eGFR. ALT vs. eGFR ( $r=-0.06, p=0.7)$; ALP vs. eGFR ( $r=0.25, p=0.2)$.

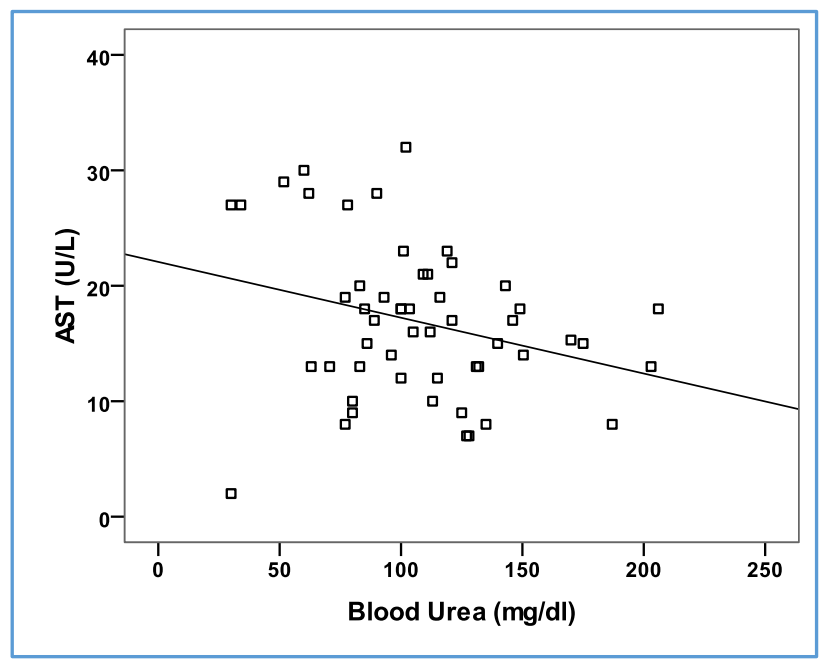

Figure 4. Correlation between Blood Urea and AST

In pre-dialysed CKD patients, AST showed a significant negative correlation with blood urea $(r=-0.285, p=0.03)$.

\section{DISCUSSION}

Haemodialysis affects various substances in the blood circulation by elevating or reducing their concentration or eliminating them. Chronic kidney disease patients undergoing haemodialysis are at higher risk of developing hepatitis $C$ infection than the general population. A study conducted in 2011 concluded that the major chronic liver diseases associated with chronic kidney disease are hepatitis B and C. ${ }^{22}$ Diagnosis and monitoring of liver damage as well as clinical management and treatment of liver disease in those patients require the continuous assessment of liver function, so we carried out this study to evaluate hepatic enzymes in order to find out possible effects of CKD upon the levels of liver function enzymes.

The present descriptive comparative study revealed significantly lower aspartate aminotransferase and alanine aminotransferase levels in haemodialysed CKD patients compared to both healthy controls and pre-dialysed CKD patients. Our results are in agreement with other studies, which revealed reduced aminotransferase levels in CKD patients undergoing haemodialysis. ${ }^{23,24}$ In accordance with our work, a study by Ray and colleagues (2015) included 100 CKD patients with ESRD, 100 CKD patients without ESRD and 100 healthy individuals showed that serum AST and ALT levels were significantly lower in CKD patients without ESRD and CKD patients with ESRD compared to controls. ${ }^{25}$ Aminotransferase levels were also significantly lower in CKD patients with ESRD compared to CKD patients without ESRD. Furthermore, a study done in Italy demonstrated lower AST and ALT levels among haemodialysed CKD patients compared to pre-dialysed CKD patients in addition to the lower level of aminotransferases has been found in both groups of CKD patients compared to healthy individuals. 26

The finding of lower aminotransferases levels CKD patients compared to pre-dialysed patients demonstrated that the decrease in aminotransferases is concomitant with the progression of renal disease. ${ }^{16}$ It was suggested that the reduction in aminotransferase enzymes in haemodialysed may be due to various factors including the elimination of aminotransferases during the HD session. The elevated lactate levels in blood, which during biochemical dosages would rapidly consume nicotinamide adenine dinucleotide phosphate (NADPH) and cause decreased aminotransferases concentration; the existence of uraemic factors that would inhibit enzyme activity; and finally decreased aminotransferase levels may result from pyridoxine deficiency, a cofactor needed for the biosynthesis of the transaminases.17,18 In accordance, Ono et al (1995) reported that patients with CKD on HD with pyridoxine deficiencies have lower serum aminotransferase levels than those without vitamin deficiencies. ${ }^{17}$ On the other hand, Lopes et al observed haemodilution and found that weight loss during HD was associated with an increase in serum concentration of ALT. The possibility of haemodilution is corroborated when the mean values of AST and ALT in the same 40 patients are compared in the samples collected before and after the HD session. There was a significant increase in both the AST and the ALT serum levels after the dialysis session. Moreover, a significant increase in the haematocrit after the HD session was also observed, which provided support for the hypothesis that patients with CKD retain water before HD. ${ }^{27,28}$ Huang et al evaluated the serum homocysteine levels of 145 patients undergoing HD and found that they were negatively related to AST. Thus, AST serum levels might reflect the high metabolic activity of homocysteine and influence its serum levels. ${ }^{29}$ Importantly, these hypotheses concern patients with CKD under HD; however, they cannot be rejected with regard to patients during pre-dialysis stages. ${ }^{28}$ 
Some researchers suggested that the aminotransferase serum levels were not related to the dialysis method. Hung et al investigated 90 patients on peritoneal dialysis and healthy adults and found an average ALT level of $15 \mathrm{IU} / \mathrm{L}$ in the peritoneal dialysis patients compared with $22 \mathrm{IU} / \mathrm{L}$ in the healthy control. ${ }^{30}$ These data are in agreement with the hypothesis that CKD patients have lower levels of ALT regardless of the dialysis method. Subsequently, some researchers have suggested that the aminotransferase serum levels could be reduced even during conservative treatment at earlier stages of CKD prior to haemodialysis treatment.26,31 In contrast to our findings, few studies have found a significant inverse relationship of AST and ALT levels with serum creatinine and a direct relationship with eGFR,26,28 observing the declining of aminotransferase levels in proportion to CKD progression.

On the contrary, alkaline phosphatase concentration was significantly higher in both groups of CKD patients than controls, and the difference between haemodialysed CKD patients and pre-dialysed CKD patients was statistically significant. Moreover, a significant negative correlation has been shown between blood urea and aspartate aminotransferase levels in CKD pre-dialysed group. On the other hand, no significant correlation has been observed between aminotransferases and duration of disease, duration of haemodialysis, eGFR or serum creatinine.

In one study, Kovesdy and his colleagues concluded that the serum ALP is an important parameter in the assessment of liver function and aids in diagnosing the type of jaundice in patients without CKD. ${ }^{19}$ However, this diagnostic importance of ALP is masked in CKD patients, as it is a well-recognised fact that serum ALP level increases in patients with CKD. In fact, in CKD patients without liver disease serum ALP can be elevated in high-turnover bone disease. ${ }^{32,33}$ In spite of this knowledge, we included ALP in our study to reiterate the non-specificity of serum ALP in CKD, further underlining the importance of correct interpretation of serum AST and ALT levels in CKD patients, more so in patients with CKD on HD. Moreover, higher levels of serum ALP are associated with increased mortality in CKD patients. ${ }^{19,34}$

In most normal individuals, approximately $95 \%$ of the total ALP activity is derived from bone and liver sources in 1:1 ratio. ${ }^{35}$ Polyacrylamide gel electrophoresis is the most reliable method of determination of the tissue origin of ALP. Bone-derived ALP is heat-labile, whereas liver-derived ALP is not. Therefore, exposure of the serum to elevated temperatures can determine the tissue source of elevated levels of ALP. Since neither polyacrylamide gel electrophoresis nor heat-testing of serum ALP is routinely used in a clinical laboratory setting, determination of the tissue source of high ALP in CKD patients with suspected hepatobiliary dysfunction is difficult.

\section{CONCLUSION}

In conclusion, serum ALT and AST levels tend to be reduced in CKD patients, particularly in those on maintenance haemodialysis treatment. Accordingly, the usage of standard normal intervals of transaminases to help detect liver disease becomes less beneficial in haemodialysis patients. These findings enforce the urgent need for establishing lower 'standard normal' reference values of liver function tests in
CKD patients in order to facilitate the diagnosis, monitoring and treatment of liver diseases, especially hepatitis C infection, an establishment that may play a role in decreasing the mortality in CKD patients.

\section{REFERENCES}

[1] Levey AS, Atkins R, Coresh J, et al. Chronic kidney disease as a global public health problem: approaches and initiatives - a position statement from Kidney Disease Improving Global Outcomes. Kidney Int 2007;72(3):247-59. http://www.kdigo.org/pdf/Levey_KI_2007.pdf

[2] Farag YM, Kari JA, Singh AK. Chronic kidney disease in the Arab world: a call for action. Nephron Clin Pract 2012;121(3-4):c120-c3. DOI: 10.1159/000345149.

[3] Levey AS, Andreoli SP, DuBose T, et al. Chronic kidney disease: common, harmful and treatable - World Kidney Day 2007. Am J Kidney Dis 2007;49(2):175-9.

[4] Levey AS, Eckardt KU, Tsukamoto Y, et al. Definition and classification of chronic kidney disease: a position statement from Kidney Disease: Improving Global Outcomes (KDIGO). Kidney International 2005;67(6):2089-100.

[5] Ofsthun NJ, LaBrecque J, Keen M, et al. The association of mortality and hospitalization with hemoglobin $(\mathrm{Hb})$ and missed dialysis treatments in stage 5 chronic kidney disease (CKD) patients with and without cardiac comorbidities. In: Nephrology dialysis transplantation. Oxford, England: Oxford University Press 2005.

[6] Alashek WA. Epidemiology of dialysis-treated endstage kidney disease in adults in Libya. PhD thesis, University of Nottingham 2013.

[7] Giannini EG, Testa R, Savarino V. Liver enzyme alteration: a guide for clinicians. Canadian Medical Association Journal 2005;172(3):367-79.

[8] Kratz A, Pesce M, Fink D. Appendix: laboratory values of clinical importance. Harrison's principles of internal medicine. 17th edn. 2012. http://www.accessmedicine. com/ content. Aspx.

[9] Fabrizi F, Poordad FF, Martin P. Hepatitis C infection and the patient with end-stage renal disease. Hepatology 2002;36(1):3-10.

[10] Kalantar-Zadeh K, McAllister CJ, Miller LG. Clinical characteristics and mortality in hepatitis C-positive haemodialysis patients: a population based study. Nephrology Dialysis Transplantation 2005;20(8):1662-9.

[11] Nakayama E, Akiba T, Marumo F, et al. Prognosis of anti-hepatitis $\mathrm{C}$ virus antibody-positive patients on regular hemodialysis therapy. Journal of the American Society of Nephrology 2000;11(10):1896-902.

[12] Fissell RB, Bragg-Gresham JL, et al. Patterns of hepatitis $\mathrm{C}$ prevalence and seroconversion in hemodialysis units from three continents: the DOPPS. Kidney International 2004;65(6):2335-42.

[13] Alashek WA, McIntyre CW, Taal MW. Hepatitis B and C infection in haemodialysis patients in Libya: prevalence, incidence and risk factors. BMC Infectious Diseases 2012;12(1): p. 265. 
[14] Rde SC, Lopea AA, Thome FS, et al. Chronic dialysis in Brazil: report of the Brazilian dialysis census, 2011. Jornal Brasileiro de Nefrologia 2012;34(3):272-7.

[15] Huraib S, al-Rashed R, Aldrees A, et al. High prevalence of and risk factors for hepatitis $\mathrm{C}$ in haemodialysis patients in Saudi Arabia: a need for new dialysis strategies. Nephrology Dialysis Transplantation 1995;10(4):470-4.

[16] De Oliveira LIR, de Almeida LEP, De Mattos CMAG, et al. Liver enzymes in patients with chronic kidney disease undergoing peritoneal dialysis and hemodialysis. Clinics (Sao Paulo) 2012;67(2):131-4.

[17] Ono K, Ono T, Matsumata T. The pathogenesis of decreased aspartate aminotransferase and alanine aminotransferase activity in the plasma of hemodialysis patients: the role of vitamin B6 deficiency. Clinical Nephrology 1995;43(6):405-8.

[18] Crawford DR, Reyna R, Weiner MW. Effects of in vivo and in vitro dialysis on plasma transaminase activity. Nephron 1978;22(4-6):418-22.

[19] Kovesdy CP, Ureche V, Lu JL, et al. Outcome predictability of serum alkaline phosphatase in men with pre-dialysis CKD. Nephrology Dialysis Transplantation 2010;25(9):3003-11.

[20] Eknoyan G, Lameire N. KDIGO 2012 clinical practice guideline for the evaluation and management of chronic kidney disease. Kidney Int 2013;3(1):1-150.

[21] Levey AS, Coresh J, Balk E, et al. National Kidney Foundation practice guidelines for chronic kidney disease: evaluation, classification and stratification. Annals of Internal Medicine 2003;139(2):137-47.

[22] Hrstić I and Ostojić R. Chronic liver diseases in patients with chronic kidney disease. Acta Med Croatica 2011;65(4):349-53.

[23] Yasuda K, Okuda K, Endo N, et al. Hypoaminotransferasemia in patients undergoing long-term hemodialysis: clinical and biochemical appraisal. Gastroenterology 1995;109(4):1295-300.

[24] Mustafa LA, Al-Abachi SB, Khalaf DS. Some biochemical changes in serum of hemodialysis patients. Natl J Chem 2008;32:695-700.

[25] Ray L, Nanda SK, Chatterjee A, et al. A comparative study of serum aminotransferases in chronic kidney disease with and without end-stage renal disease: need for new reference ranges. International Journal of Applied and Basic Medical Research 2015;5(1):315 .
[26] Fabrizi F, Lunghi G, Finazzi S, et al. Decreased serum aminotransferase activity in patients with chronic renal failure: impact on the detection of viral hepatitis. American Journal of Kidney Diseases 2001;38(5):1009-15.

[27] Lopes EP, Sette LH, Sette JB, et al. Serum alanine aminotransferase levels, hematocrit rate and body weight correlations before and after hemodialysis session. Clinics (Sao Paulo) 2009;64(10):941-5.

[28] Sette LHBC, de Almeida LEP. The reduction of serum aminotransferase levels is proportional to the decline of the glomerular filtration rate in patients with chronic kidney disease. Clinics (Sao Paulo) 2015;70(5):346-9.

[29] Huang JW, Yen CJ, Pai Mf, et al. Association between serum aspartate transaminase and homocysteine levels in hemodialysis patients. American Journal of Kidney Diseases 2002;40(6):1195-201.

[30] Hung KY, Lee KC, Yen CJ, et al. Revised cut-off values of serum aminotransferase in detecting viral hepatitis among CAPD patients: experience from Taiwan, an endemic area for hepatitis B. Nephrology Dialysis Transplantation 1997;12(1):180-3.

[31] Sette LH, de Almeida LEP. Liver enzymes serum levels in patients with chronic kidney disease on hemodialysis: a comprehensive review. Clinics (Sao Paulo) 2014;69(4):271-8.

[32] Magnusson P, Sharp CA, Magnusson M, et al. Effect of chronic renal failure on bone turnover and bone alkaline phosphatase isoforms. Kidney International 2001;60(1):257-65.

[33] Torres PU. Bone alkaline phosphatase isoforms in chronic renal failure. Kidney International 2002;61(3):1178-9.

[34] Beddhu S, Ma X, Baird B, et al. Serum alkaline phosphatase and mortality in African Americans with chronic kidney disease. Clinical Journal of the American Society of Nephrology 2009;4(11):1805-10.

[35] Magnusson P, Degerblad M, Saaf M, et al. Different responses of bone alkaline phosphatase isoforms during recombinant insulin-like growth factor-I (IGF-I) and during growth hormone therapy in adults with growth hormone deficiency. Journal of Bone and Mineral Research 1997;12(2):210-20. 